\title{
Introspección reflexiva del estudiante sobre su experiencia en másteres internacionales
}

\author{
Students' Reflective Statements about their experience in International Master programs
}

\author{
Martina G. Gallarza \\ Universitat de València \\ martina.gallarza@uv.es \\ Teresa Fayos Gardó \\ Universitat de València \\ teresa.fayos@uv.es \\ María Fontana Vinat \\ Universitat de València \\ mariafontanavinat@gmail.com \\ Belén Derqui Zaragozá \\ IQS, Universitat Ramon Llull \\ belen.derqui@iqs.edu
}

Fecha presentación: 08/11/2018 | Aceptación: 17/12/2018 |Publicación: 21/12/2018

\begin{abstract}
Resumen
El estudiante universitario juega hoy un papel activo no solo como cliente, sino como co-creador de valor. Con una técnica de investigación cualitativa como la autorreflexión personal, se puede lograr su participación y co-creación mediante una introspección subjetiva. Este trabajo explora el valor percibido de la experiencia universitaria a través de 30 introspecciones reflexivas de estudiantes de Másteres internacionales con el apoyo de un CAQDAS. Los resultados, explicados en relación con los beneficios y costes, y con las dimensiones de valor percibido de la experiencia (funcional, emocional y social), muestran la utilidad de la introspección para la comprensión de la experiencia universitaria intercultural y multidimensional.
\end{abstract}

Palabras clave: Educación Superior; introspección reflexiva; diferencias interculturales; dimensiones de valor.

\section{Resum}

L'estudiant universitari juga avui un paper actiu no només com a client, sinó com a co-creador de valor. Amb una tècnica d'investigació qualitativa com l'autoreflexió personal, es pot aconseguir la seva participació i co-creació mitjançant una introspecció subjectiva. Aquest treball explora el valor percebut de l'experiència universitària a través de 30 introspeccions reflexiva d'estudiants de màster internacionals amb el suport d'un CAQDAS. Els resultats, explicats en relació amb els beneficis i costos, i amb les dimensions de valor percebut de l'experiència (funcional, emocional i social), mostren la utilitat de la introspecció per a la comprensió de l'experiència universitària intercultural i multidimensional.

Paraules clau: gamificació; Educació Superior; introspecció reflexiva; diferències interculturals; dimensions de valor.

\section{Abstract}

Value co-creation is becoming a mainstream in higher education, as a progress within the 'Students as Customer' approach. Furthermore, the development of qualitative studies through reflective methods from students can help instructors and managers. The aim of this research is to explore with the aid of a CAQDAS, 30 students' reflective statements in two master programs. Findings are presented in terms of value dimensions: functional, emotional and social, and in terms of positive (benefits) and negative (cost) aspects of the experience. The usefulness of introspective methodologies from students is shown in the evaluation of the multidimensional and intercultural educational experience.

Key Words: Higher Education; Reflective Statements; Intercultural Differences; Value Dimensions. 
Gallarza, M.G., Fayos Gardó, T., Fontana Vinat, M., Derqui Zaragozá, B. (2018). Introspección reflexiva del estudiante sobre su experiencia en másteres internacionales. @tic revista d'innovació educativa, 21, 57-66.

\section{Introducción}

Dos son las orientaciones metodológicas que han dominado las Ciencias Sociales, entendidas como dos paradigmas metodológicos básicos (Deshpandé, 1983): el paradigma cuantitativo y el cualitativo. Sin embargo, en Marketing, algunas voces se han alzado en contra de un excesivo uso del primero en detrimento del segundo (Gummesson, 2005). Cuando la experiencia de consumo a evaluar es compleja, subjetiva, de difícil aprehensión y multidimensional, las aproximaciones cualitativas cobran más sentido. Este es el caso de los estudiantes universitarios, cuando evalúan su propia experiencia, multifacética y altamente personal (Ledden et al., 2007; Gallarza et al., 2017), y que puede ser por tanto analizada mediante el análisis del propio discurso de los estudiantes como consumidores (Sitz, 2008).

Este trabajo busca explorar la experiencia de estudiantes internacionales mediante el análisis de sus ejercicios de redacción de reflective statements. Esta técnica, perteneciente a los métodos de investigación cualitativa (Patton, 2002) y más concretamente a las técnicas propias o teoría de la interpretación (O'Shaughnessy y Holbrook, 1988; 0`Shaughnessy, 2009), se basa en la introspección para despertar sentimientos, ideas y actitudes (Smith, 2016). Con esta metodología se activa la participación del estudiante en su experiencia y en su evaluación, en línea con la llamada co-creación de valor (Leclerc et al., 2016) que propugna una mayor participación del consumidor en el servicio, que en educación, daría lugar a la idea de que "los estudiantes han empezado a mostrar interés por adoptar roles activos y participativos que les permitan interactuar $y$ trabajar colaborativamente con los educadores" (Dollinger et al., 2018). A esta oportunidad metodológica se añade una circunstancia actual, las diferencias interculturales en los procesos de aprendizaje, que obliga a una nueva comprensión del servicio de educación universitaria (Fan et al., 2012; Kaur y Noman, 2015; Rienties et al., 2014; Strauss et al., 2014).

Al encuentro de estas dos tendencias (investigación introspectiva y capacidad de co-creación en el aula), planteamos este trabajo que tiene como principal objetivo, explorar la utilidad de los reflective statements como herramienta a utilizar en los masters internacionales de nuestras universidades, para una mejor comprensión de los beneficios y costes derivados de la interculturalidad de los estudiantes. La consecución de dicho objetivo, permitirá la consecución de otro complementario: analizar el contenido de reflexiones particulares de los estudiantes respecto a su experiencia universitaria, mostrando la riqueza del valor de la experiencia.

El análisis de beneficios y costes puede hacerse desde la óptica del valor percibido, en la medida en que dicho valor es una medida multidimensional de la experiencia de consumo (Holbrook, 1999; Swenney y Soutar, 2001), como evaluación global de elementos positivos y negativos de la experiencia (Zeithaml, 1988). Dicha aproximación ya se ha utilizado en el análisis de la experiencia de educación superior, tanto con técnicas cualitativas (Li et al., 2016; Gallarza et al., 2017) como cuantitativas (Ledden et al., 2007). Metodologicamente, para lograr los objetivos propuestos, este trabajo plantea un análisis de contenido realizado sobre reflective statements de 30 estudiantes de dos Master internacionales interuniversitarios en la Universidad de Valencia. El análisis de contenido se realizó a través de un CAQDAS o software de Análisis de Datos Cualitativos Asistido por Computadora.

\section{Marco Conceptual \\ 2.1 El uso de la investigación cualitativa reflexiva en educación}

El uso de metodologías reflexivas en la educación se entronca en los debates epistemológicos de las Ciencias Sociales, en las que, el positivismo ha sido una postura dominante frente a la investigación de corte fenomenológico y/o "interpretativista" que postula la variedad y combinación de metodologías en pro de un mejor equilibrio entre los dos estados, que Denzin (2001, p. 325) define como: "el objetivo, políticamente neutral observador que permanece fuera y por encima del estudio“, y el del investigador interpretativo, que "está histórica y localmente situado en el corazón de los varios procesos estudiados".

El uso de metodologías reflexivas en educación es controvertido (Klenowski y Lunt, 2008; Mena-Marcos et al., 2013; Mitchell, 2013), al referir también la idea de una implicación y falta de objetividad del evaluador. Sin embargo, se han utilizado enfoques cualitativos en el proceso de enseñanza aprendizaje, que han permitido producir un aprendizaje más innovador, donde la creatividad se valora tanto como la entrega "clásica" de conocimiento, con resultados positivos para estudiantes y docentes (Klein et al., 2007). En este sentido, "se espera que los estudiantes en Educación Superior participen activamente en el pensamiento crítico y la creación de conocimiento, en lugar de la recepción pasiva de un conocimiento dado" (Lee et al., 2015: p.31). El objetivo de las actividades de prácticas reflexivas es el fomento del aprendizaje profundo y la preparación de los estudiantes para las demandas profesionales, mediante el aprendizaje colaborativo en grupos (Jackling et al., 2015). En definitiva "el objetivo final de cualquier programa que pretenda desarrollar la reflexión no ha de ser otro que el de fomentar en los futuros profesionales habilidades metacognitivas que les permitan interrogar, analizar, conocer, evaluar y modificar su propia práctica desde un punto de vista profesional, moral y social" (Moya et al., 2010: p.13). Así, el uso de las técnicas reflexivas está en clara consonancia con los objetivos del EEES, al fomentar habilidades para un aprendizaje social, y a lo largo de la vida.

No obstante, utilizar metodologías de reflexión en el aula también requiere profesores que actúen como intelectuales críticos y reflexivos (Bain, 2006), capaces de "utilizar su conocimiento cultural, disciplinar $y$ pedagógico para analizar y examinar críticamente su propia actividad práctica y su manera de razonar en la disciplina" (Moya et al., 2010: p.12). La literatura muestra cómo el pensamiento reflexivo está relacionado positivamente con la inteligencia emocional y la capacidad de comunicar (Afshar y Rahimi, 2016). Se busca con estas técnicas, explorar, descubrir y aceptar la perspectiva de los demás y dudar de las propias razones. De este proceso surge la crítica reflexiva imprescindible además en nuestras aulas actualmente, en donde suele existir una convivencia intercultural (Ciges, 2012).

Entre las formas de adoptar este enfoque cualitativo en el aula, el uso de la autorreflexión personal es verdaderamente innovador (Smith, 2016), tomando la forma de reflective statements cuyo uso en educación ha demostrado ser, al mismo tiempo, útil y difícil (Klenowski 
Gallarza, M.G., Fayos Gardó, T., Fontana Vinat, M., Derqui Zaragozá, B. (2018). Introspección reflexiva del estudiante sobre su experiencia en másteres internacionales. @tic revista d'innovació educativa, 21, 57-66.

y Lunt, 2008; Mena-Marcos et al., 2013; Mitchell, 2013). Esta técnica permite a los individuos explorar introspectivamente los sentimientos, las actitudes y el conocimiento expresándolos libremente en forma de escritos, imágenes, videos, etc. Se centra en la premisa de que el conocimiento está continuamente sujeto a cambios como resultado de capacidades activas y reflexivas. El uso de dicha autorreflexión personal debe confluir con la idea de que como docentes e investigadores universitarios hay que ser capaces de usar un "pensamiento crítico (que) implica dominar las habilidades cognitivas, como la interpretación, el análisis y la evaluación" (Dahl et al., 2018: p.102). Más específicamente, las autorreflexiones de los estudiantes "pueden documentar el aprendizaje significativo que es evaluable como una forma creativa de autoexpresión" (Smith, 2016: p.1).

Respecto al uso de estas metodologías en la docencia de Marketing, existe poca literatura. Dahl et al. (2018) realizan una perspectiva histórica del pensamiento crítico y aprendizaje reflexivo en la literatura de educación en Marketing, concluyendo que: "Los investigadores deben seguir identificando maneras de desarrollar el pensamiento crítico y las habilidades de aprendizaje reflexivo en nuestros estudiantes" (p. 12). Los docentes en Marketing, al no pertenecer al campo del conocimiento de la psicología o la pedagogía, estamos en inferioridad de condiciones respecto del uso de estas metodologías más innovadoras en el aula, pero nuestra principal motivación para emprenderlas debe ser la idea general de que, con el pensamiento reflexivo, la experiencia siempre genera algo de aprendizaje.

En definitiva, para promover un aprendizaje reflexivo es imprescindible potenciar el pensamiento crítico siguiendo las normas de una disciplina, pero al mismo tiempo superando sus límites, aceptando y reconociendo la interdisciplinariedad (Moya et al., 2010). Así, el docente en Marketing que utilice estas técnicas está combinando docencia e investigación, puesto que puede aplicar en su aula un conocimiento sobre metodologías propias del ámbito más fenomenológico del estudio del comportamiento del consumidor, y con ello también conseguir co-crear mayor valor para todas las partes implicadas.

\subsection{Efectos de la interculturalidad en la experiencia universitaria}

Como telón de fondo de nuestro trabajo está la realidad del tema de la interculturalidad en las universidades, que ha sido tratado desde diversas perspectivas: las posibles motivaciones para estudiar en el extranjero (Alfaro et al., 2009), las causas del incremento de la movilidad (Rivza y Teichler, 2007), la influencia de la cultura nacional en las preferencias de estilo de aprendizaje (Kelly, 2009), las diferencias culturales en la comunicación de retroalimentación con el alumnado (Tian y Lowe, 2013), o las barreras en grupos interculturales, (Rafferty 2013). Todos estos trabajos, aunque en distintos contextos geográficos y disciplinares, apuntan hacia el aprovechamiento de una mayor movilidad y creación de grupos multiculturales y, por tanto, a la búsqueda de las implicaciones de este fenómeno en las aulas, en tanto en cuanto afecta al proceso de aprendizaje (Haug, 2016).

Sin embargo, la literatura que estudia los efectos que tiene la cultura sobre la enseñanza y el aprendizaje no es unánime ni en sus resultados, ni en el enfoque. Algunas investigaciones, demostraron que la cultura tiene un efecto no univoco, en la selección de las metodologías de enseñanza para los docentes (Kaur y Noman, 2015). Otros encontraron diferencias entre estudiantes de master de Europa occidental y oriental, no europeos y asiáticos, respecto a sus niveles de integración académica y social (Rienties et al., 2014). Mientras que otros, estudiaron el cambio positivo en las percepciones de los estudiantes nacionales e internacionales sobre la influencia de la diversidad grupal en la comunicación, el aprendizaje, el rendimiento y los grados de evaluación, antes y después de haber trabajado en un grupo intercultural (Moore y Hampton, 2015). Así, las consecuencias de un grupo diverso se pueden manifestar tanto en beneficios del proceso cognitivo como en dificultades de integración (Kelly, 2009).

En definitiva, los efectos de la cultura en el proceso de aprendizaje son difíciles de aprehender, ya que entran en juego otros factores más allá de la cultura (Lee et al., 2015): las disciplinas, el lenguaje, la enseñanza preuniversitaria, la diversidad, la personalidad, el género, la capacidad individual y el tamaño del grupo. En este sentido, la interculturalidad favorece las políticas de orientación activa hacia las necesidades del estudiante como consumidor y co-creador de valor en la enseñanza universitaria.

\subsection{La orientación al estudiante como cliente y la co- creación de valor}

La internacionalización de la Universidad lleva también aparejado el incremento de las expectativas y exigencias sobre la calidad de los servicios de Educación Superior. La llamada orientación Student as Customer (SAC), aparecida en los 90 en las Universidades británicas (Bunce et al., 2016), propugna la satisfacción de los estudiantes como clientes y el desarrollo de estrategias de fidelización como imperativo de las universidades (Finney y Finney, 2010). Los estudiantes son el principal cliente interno que genera una lealtad afectiva en forma de comunicaciones positivas (De La Fuente et al., 2010). Bajo el enfoque SAC, se espera que los estándares y la calidad del servicio aumenten porque los clientes tienen control sobre las expectativas y evalúan los servicios por su capacidad para satisfacer sus demandas, lo que es un beneficio, tanto para el estudiante como consumidor como para otros stakeholders, en la búsqueda de la excelencia en el sistema educativo. Sin embargo, existen posiciones no tan optimistas que sugieren que la postura SAC puede convertirse en una preocupación para las universidades por su tendencia hacia el clientelismo (Bunce et al., 2017; Swensson y Wood, 2007). En cualquier caso, esta realidad obliga a pensar en un marketing educativo que entiende y se preocupa por los estudiantes como co-creadores de valor, y que debe encontrar nuevas metodologías (Gibbs, 2007) para orientarse hacia dicha co-creación (Dollinger et al., 2018).

Así, con este enfoque SAC, y para una mayor co-creación de valor, los estudiantes pueden como consumidores de servicios educativos, combinar sus cuatro roles diferentes (Guolla, 1999): como consumidores, como clientes, como productores y como productos del servicio docente. Esta multiplicidad de roles está en la idea original de co-creación de valor (Prahalad y Ramaswamy, 2004), que permite reinterpretaciones en las múltiples aproximaciones posteriores a dicho concepto de cocreación, todas ellas en la línea de la colaboración e interactividad (Leclerq et al., 2016).

Como muestra la Figura 1, el enfoque de este trabajo 
Gallarza, M.G., Fayos Gardó, T., Fontana Vinat, M., Derqui Zaragozá, B. (2018). Introspección reflexiva del estudiante sobre su experiencia en másteres internacionales. @tic revista d'innovació educativa, 21, 57-66.

bebe de esta nueva realidad, de forma que la aplicación de métodos de investigación cualitativa reflexivos y propios de la introspección reflexiva en estudiantes miembros de un grupo multicultural, puede ser una forma de analizar esta co-creación de valor, dentro de la orientación SAC. El estudiante, co-creador de valor (como usuario final del servicio de la universidad), a través del proceso de auto-reflexión personal, contribuye a la retroalimentación del sistema y puede ser evaluado en la medida en que participa en dicho proceso de cocreación.

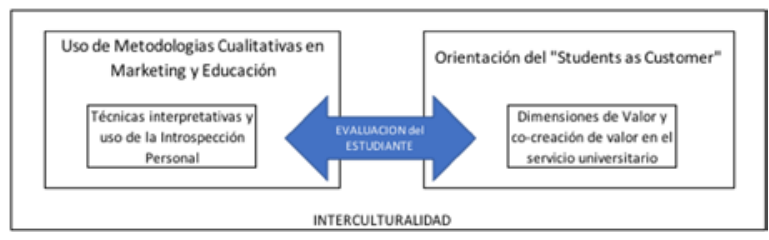

Figura 1. Planteamiento interdisciplinar y metodológico.

\section{Trabajo empírico}

El objeto de estudio de nuestro análisis cualitativo lo componen 30 reflective statements realizados por estudiantes de dos masters en la Universidad de Valencia; MBA internacional, iMBA, (Facultad de Economía) y WOP-P (Facultad de Psicología). Los reflective statements fueron redactados al término de sendas asignaturas de Marketing en sendas titulaciones (curso 2015-2016).

Ambos programas pertenecen a alianzas de varias Universidades (EE.UU., Reino Unido, Francia, Rusia, Alemania, Malasia y España para el programa iMBA, e Italia, Francia, Portugal y España para el programa WOPP) por lo que los estudiantes tienen diferentes orígenes y nacionalidades. Los graduados en ambos masters obtienen una doble titulación: un grado otorgado por la universidad donde completaron sus módulos principales, y otro por la universidad donde se especializaron y terminaron su master Tesis.

El reflective statement es parte de la evaluación final del alumno, relacionada con los resultados de aprendizaje, especificados en el plan de estudios de ambas materias como: "Como parte de su tarea, se puede obtener hasta un 5\% de la calificación mediante la redacción de un reflective statement de carácter individual, realizado al final del trabajo en grupo, que debe versar sobre los siguientes aspectos: debilidades

Lo que has aprendido sobre tus fortalezas y

Lo que has aprendido como miembro del equipo de tu trabajo

¿Qué harías de manera diferente como resultado de la experiencia del equipo?

¿Cómo crees que aplicas el aprendizaje de esta experiencia a tu vida laboral?".

Adoptando un enfoque SAC, los estudiantes son considerados consumidores de servicios de educación superior que han obtenido un valor de su experiencia, por lo que las valoraciones contenidas en los 30 documentos pueden considerarse valores percibidos de la experiencia educativa (Holbrook, 1999). En consecuencia, siguiendo la literatura sobre valor percibido (Zeithaml, 1988), se asumió un enfoque en dos pasos para codificar y etiquetar las dimensiones de la experiencia. En primer lugar, dada la naturaleza multidimensional del valor, y en consonancia con la literatura (Sheth et al., 1991; Sweeney y Soutar, 2001), se consideraron tres dimensiones en las valoraciones de los estudiantes: funcional, emocional y social. En segundo lugar, estas experiencias pueden ser positivas o negativas ya que se solicita a los estudiantes que expresen sus fortalezas y debilidades. Así, los comentarios positivos y negativos son ejemplos de una experiencia de preferencia, adoptando por tanto un enfoque de trade-off (beneficios menos costes), como forma bien conocida de conceptualizar el valor en el comportamiento del consumidor (Zeithaml, 1988).

Dado que la estructura de los dos master es interuniversitaria, y algunos de los resultados de aprendizaje especificados en las guías docentes se relacionan con la capacidad de trabajar en equipos interculturales, su naturaleza es idónea, para comentar exploratoriamente los resultados en las diferencias interculturales, en un nivel complementario, después del enfoque positivo frente al negativo (costes y beneficios), y la distinción funcional, emocional y social, propios de la literatura sobre valor (Holbrook, 1999; Sheth, Newman y Gross, 1991; Sweeney y Soutar, 2001; Zeithaml, 1988). Dada la naturaleza exploratoria del objetivo de este estudio, el enfoque cualitativo se basa en "comprender fenómenos complejos conociendo los comportamientos o ideas de personas $u$ organizaciones sociales involucradas, así como sus valores, rituales, símbolos, creencias y emociones" (Gal y Ograjenšek, 2010: p.1). Según lo propuesto por estos autores y otros como Patton (2002), los enfoques cualitativos interpretan la perspectiva social a través de hallazgos que no son estadísticos: en nuestro caso, la documentación disponible es objeto de un análisis de contenido, utilizando, tal y como sugieren Sinkoviks \& Alfoldi (2012), un software CAQDAS de análisis de datos cualitativos (Atlas-ti 7.5.10), y siguiendo una serie de pasos.

En primer lugar, a cada uno de los 30 documentos, se le asignó un código que incorporó información adicional sobre el género y la nacionalidad de los estudiantes (Tabla 1).

\begin{tabular}{|c|c|c|c|}
\hline \multicolumn{2}{|c|}{ Programa de Master iMBA UV } & \multicolumn{2}{|c|}{ Programa de Master WOP-P UV } \\
\hline Estudiante & Nacionalidad y Género & Estudiante & Nacionalidad y Género \\
\hline 101.M.00 & España, Hombre & W01.F.72 & Brasil, Mujer \\
\hline I02.F.31 & Líbano, Mujer & W02.F.03 & Italia, Mujer \\
\hline 103.M.00 & España, Hombre & W03.F.20 & Serbia, Mujer \\
\hline 104.F.00 & España, Mujer & W04.F.03 & Italia, Mujer \\
\hline 105.F.00 & España, Mujer & W05.M. 80 & Islas Australasia, Hombre \\
\hline 106.F.00 & España, Mujer & W06.F.20 & Serbia, Mujer \\
\hline 107.M.51 & Libia, Hombre & W07.F.72 & Brasil, Mujer \\
\hline 108.M.01 & Bélgica, Hombre & W08.F.03 & Italia, Mujer \\
\hline I09.F.70 & Ecuador, Mujer & W09.F.03 & Italia Mujer \\
\hline I10.M.52 & Marruecos, Hombre & W10.M.04 & Francia, Hombre \\
\hline I11.F.70 & Ecuador, Mujer & W11.M.03 & Italia, Hombre \\
\hline |12.F.02 & Bulgaria, Mujer & W12.M.03 & Italia, Hombre \\
\hline I13.M.00 & España, Hombre & W13.M.03 & Italia, Hombre \\
\hline |14.F.71 & Honduras, Mujer & W14.F.04 & Francia, Mujer \\
\hline & & W15.M.05 & Portugal, Hombre \\
\hline & & W16.F.05 & Portugal, Mujer \\
\hline
\end{tabular}

Tabla 1. Perfil de los participantes.

En el proceso de análisis del contenido de la información, se buscó por temas recurrentes o centrales tal y cómo aconseja Patton (2002), por lo que la segunda fase consistió en identificar, codificándolos, los párrafos en los que se hablaba de alguno de los seis elementos pre-identificados en la literatura (beneficios y/o costes funcionales, emocionales y sociales). En la tercera fase, cuando el número de citas asignadas a cada elemento superaba el umbral de 25 (previamente acordado por los investigadores) se descomponían en categorías, e incluso subcategorías, para las que se creaban nuevas codificaciones. Tres investigadores participaron en el proceso de codificación del contenido de las entrevistas, 
Gallarza, M.G., Fayos Gardó, T., Fontana Vinat, M., Derqui Zaragozá, B. (2018). Introspección reflexiva del estudiante sobre su experiencia en másteres internacionales. @tic revista d'innovació educativa, 21, 57-66.

tanto de forma individual, como posteriormente de forma colectiva (Miles \& Huberman, 1994) en la discusión de nuevos temas y códigos ausentes en la literatura previa.

\section{Análisis y discusión de Resultados}

La Tabla 2 recoge el número de citas identificadas en la investigación organizadas en categorías y subcategorías. El primer resultado general, de la investigación exploratoria realizada, es lo positiva que resultó la experiencia: se codificaron 176 beneficios y solo 23 costes. Este resultado de un balance positivo beneficios/costes es similar al cualitativo de Moore y Hampton (2015) sobre la percepción de los estudiantes de la evaluación de grupos interculturales, siendo los comentarios de los beneficios percibidos similares a las razones mencionadas por ellos.

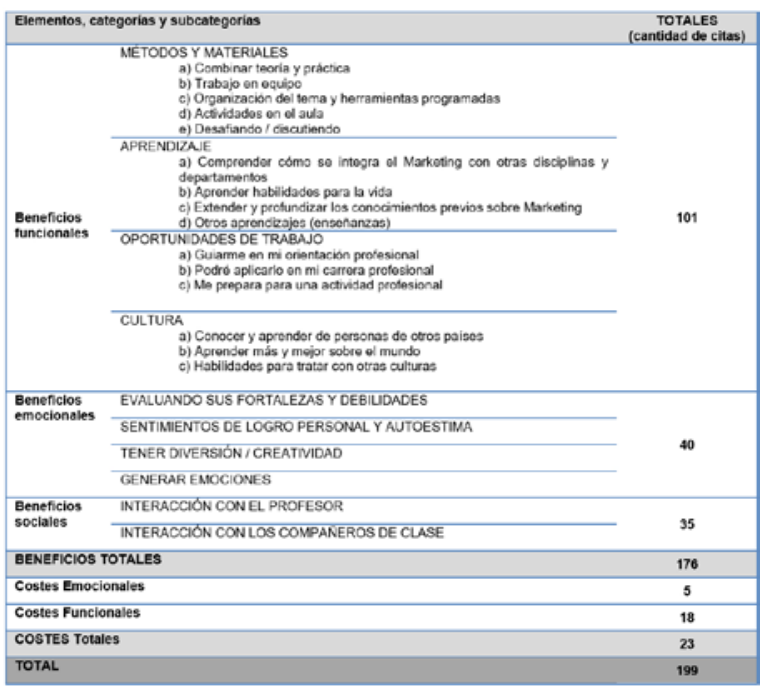

Tabla 2. Número de citas identificadas como beneficios o costes en el análisis de contenido.

Fuente: Elaboración propia.

\subsection{Beneficios Funcionales}

La primera categoría de beneficios funcionales identificada fue MÉTODOS Y MATERIALES; Ios análisis de contenido revelaron cinco subcategorías: (a) la mayoría de los estudiantes mencionó la combinación de teoría y práctica, independientemente del master, género o nacionalidad del alumno, subrayando su carácter realista y enriquecedor. Los estudiantes expresaron sentimientos de aprobación, combinando emociones y conocimientos, en el proceso de aprendizaje. Finalmente, como se pretendía, el ejercicio dio retroalimentación al docente sobre métodos y herramientas: W15: "una representación gráfica y ejemplos de la vida real fue fundamental para que yo entendiera la clase $y, . . . c r e o$ que esta es una herramienta fundamental para un docente y nos involucra, nos organiza la información y facilita la comprensión y la memorización de los temas". La segunda subcategoría fue el (b) Trabajo en equipo, actividad esperada por los estudiantes, y verdaderamente positiva: 114: "Otro punto a destacar para mí fue que se nos exigía que trabajáramos en grupos en la mayoría de los ejercicios, algo que esperaba del iMBA, pero se hizo de una manera original". Este aspecto positivo es similar a lo planteado hace casi una década por Kelly (2009), en donde a largo plazo, una vez superado un periodo de adaptación determinado, el resultado del trabajo en grupos interculturales suele ser más eficiente que el de los grupos monoculturales.

Otras subcategorías identificadas están estrechamente relacionadas unas con otras, asumiendo cierta subjetividad en el proceso de categorización como es habitual en el análisis de contenido (Smith, 2016): (c) organización del tema y herramientas programadas, W06: "Encontré el material fácil de entender y se explicó en la primera clase que cada uno de nosotros ya tiene algún conocimiento sobre marketing porque cada uno de nosotros es un consumidor"; (d) Actividades en el aula y (e) Desafiando /discutiendo: I02: "El material era muy desafiante pero aplicable a las actividades diarias al mismo tiempo, lo que lo hace más accesible, fácil de relacionar con el material".

En cuanto a los beneficios funcionales relacionados con el Proceso de APRENDIZAJE, Los estudiantes de WOP-P fueron casi unánimes en señalar el valor desconocido de estudiar Marketing, I03: "Después de realizar el curso, en mi mente el marketing ya no es solo comunicación y publicidad. Es mucho más que eso". En cuanto a la interculturalidad, esta categoría de APRENDIZAJE fue mencionada solo por estudiantes de Europa y América Latina. Los beneficios de esta categoría se reagruparon en cuatro categorías: (a) Darse cuenta de cómo el marketing se integra con otras disciplinas $y$ departamentos, que principalmente para los estudiantes WOP-P, escapa a los estereotipos, dejando de considerarla un área poco importante y pasando a considerarla una parte central del negocio de la empresa que afecta a toda. (b) Aprendizaje de habilidades para la vida: ya que les facilitó poder apreciar mejor el mundo en el que viven desde la perspectiva del marketing. (c) Extender y profundizar los conocimientos previos sobre Marketing, superando la frecuente concepción del marketing de exclusivamente comunicación y publicidad que tienen los no iniciados. Y (d) Otras enseñanzas, que contiene comentarios más comprensivos como: W07: "el proyecto final, que nos desafió a pensar globalmente sobre todas las etapas importantes para elaborar un plan de negocios, integrando todas las ideas y la información en las que cada miembro estaba trabajando, realmente trabajando en equipo".

Al igual que sucedía para MÉTODOS Y MATERIALES, las diferencias entre los dos grupos de estudiantes son notables, ya que el aprendizaje sobre la integración del Marketing como disciplina y como función comercial es más común en los estudiantes de WOP-P, I02: "Finalmente, con los enriquecidos conceptos que he encontrado a lo largo del curso, aprendí que el marketing es un canal abierto de comunicación entre dos partes que se benefician", aunque también se menciona por parte de los estudiantes de IMBA I04: "ser conscientes de cómo las empresas cambian las estrategias y suponer que podría reconocerlo. Como estudiante de negocios espero de mí mismo que al ver estas cosas comprenda qué hay dentro de esos cambios. Es algo que debo mejorar y probablemente no evitar los anuncios sería una buena manera de comenzar".

La tercera subcategoría son las OPORTUNIDADES DE TRABAJO. En este caso, la categorización responde al criterio subjetivo de los autores, aunque expresiones similares respecto a la mejora de las capacidades profesionales de los estudiantes también se recogen en anteriores trabajos (Mitchell, 2013). Codificamos las 12 afirmaciones, que en su mayoría fueron citadas por los estudiantes de WOP-P, en tres categorías: (a) Guiándome 
Gallarza, M.G., Fayos Gardó, T., Fontana Vinat, M., Derqui Zaragozá, B. (2018). Introspección reflexiva del estudiante sobre su experiencia en másteres internacionales. @tic revista d'innovació educativa, 21, 57-66.

en mi orientación profesional, ya que les abrió la mente sobre amplias posibilidades de la disciplina y reduciendo los frenos internos de su capacidad de desarrollarla; (b) Podré aplicarlo en mi carrera profesional: I12: "Teniendo en cuenta mi orientación hacia las empresas multinacionales, espero poder aplicar las herramientas técnicas aprendidas en el tema"; Y (c) Me prepara para una actividad profesional, sobre todo gracias a las actividades prácticas realizadas a lo largo de la asignatura. Esta percepción sobre la preparación profesional adquirida está en línea con el enfoque de Warwick (2014) en el ámbito del desarrollo de estrategias de internacionalización de las universidades. La última categoría de beneficios funcionales está relacionada con la CULTURA. Aunque los aspectos interculturales son difíciles de aprehender independientemente de otros temas, tal y como se esperaba de acuerdo con la naturaleza y organización de los dos Master, la cultura tenía que estar patente en los estudiantes al hacer una introspección sobre su experiencia. La categoría se dividió en tres subcategorías: (a) Conocer y aprender de personas de otros países. Muchos estudiantes mencionaron como muy interesante, motivadora, gozosa e integradora una actividad en clase donde los estudiantes deben presentar de una forma creativa su propio país como un producto, indicando I14 que: "Aprendimos muchas cosas nuevas sobre otros lugares, pero también sobre cada uno, con lo que pensé que era una buena actividad de integración (entender por qué somos como somos, diferencias y similitudes)". (b) Aprender más y mejor sobre el mundo, fue mencionado por ambos grupos, y se debió sobre todo a la multiculturalidad del grupo que les aportó una visión de primera mano más amplia sobre el mundo, sorprendiéndoles percatarse con una orientación muy positiva, de sus similitudes y diferencias. $Y$ (c) Habilidades para tratar con otras culturas, fueron mencionadas en ambos programas: I04: "la mayoría de nosotros hemos aprendido a lidiar con las diferencias culturales".

\subsection{Beneficios emocionales}

Entre los resultados de este ejercicio también surgieron beneficios emocionales, aunque con menor frecuencia que los funcionales. Se consideraron "emocionales" expresiones que mencionasen los sentimientos internos y una evaluación personal y psicológica. Muchas afirmaciones son de naturaleza híbrida dual funcional/emocional, y también involucran, aspectos de relación e integración social. La clasificación fue por tanto, más difícil y subjetiva que en los beneficios funcionales, obteniéndose 4 categorías.

En la categoría EVALUANDO FORTALEZAS Y DEBILIDADES, los estudiantes identificaron sus fortalezas ya existentes, y las nuevas fortalezas o roles que estaban desarrollando. También cómo su rasgo de personalidad les hizo darse cuenta de las debilidades, W14: "Mi timidez todavía es una debilidad, pero con presentaciones orales y diversas interacciones en clase, aprendí a luchar un poco con eso". Muchos mencionaron también la capacidad de trabajar bajo presión, e incluso hubo para quien pasó de ser una debilidad a una fortaleza.

En la categoría SENTIMIENTOS DE AUTOREALIZACIÓN Y AUTOESTIMA, los estudiantes mencionaron la idea de un "desafío" en su experiencia de master, tanto explícitamente, como implícitamente W16: “(...) terminé siendo el primero y me sentí muy feliz y emocionado con el producto que desarrollé. (...) y esto sucedió porque estaba muy motivado y comprometido con el proyecto". Se consideró la categoría DIVERSIÓN / CREATIVIDAD ya que muchos estudiantes afirmaron haber sido capaces de desarrollar la creatividad y divertirse en clase y fuera de clase, lo que corresponde a lo que Sheth et al. (1991) acuñaron como "valor epistémico" en una categoría precisa de beneficios emocionales. W02: “(...) no eran proyectos aburridos, sino que realmente nos desafiaban a ser creativos, innovadores y curiosos, y al mismo tiempo nos permitían aprender mucho". En conjunto, en línea con el valor inesperado derivado de la asignatura (epistémico), algunos estudiantes se expresaron de una manera más general: 108: "Este master realmente me convenció como elección. Me aportó nuevas ideas y nuevas perspectivas de futuro".

Por último, la categoría GENERAR EMOCIONES POSITIVAS contiene citas con sentimientos más generales, en ocasiones en forma de adjetivos (por ejemplo, estimulante, refrescante, placentero) expresados para actividades precisas realizadas a lo largo de la asignatura Marketing, o de forma general: W15: “(...) me gustaría concluir diciendo que esta fue una experiencia increíblemente agradable".

\subsection{Beneficios sociales}

De acuerdo con la naturaleza multidimensional del valor (Holbrook, 1999; Sweeney y Soutar, 2001), los beneficios sociales también se identificaron como otra dimensión de la experiencia. En consonancia con otros trabajos sobre valor percibido de experiencias de educación en interculturalidad (Gallarza et al., 2017) se identificaron dos categorías, INTERACCIÓN CON EL PROFESOR E INTERACCIÓN CON LOS COMPAÑEROS DE CLASES, aunque algunos estudiantes relacionaron ambas, en general, 108: "el espíritu de la clase fue tan agradable, gracias a mis grandes colegas, los profesores eran realmente interesantes y motivados", o para momentos precisos, W11: "Me gustó mucho discutir con el profesor y compañeros de clase sobre la ética detrás de los anuncios, cuáles son buenos y cuáles son malos". La vinculación con el profesor se mencionó en relación con el compromiso personal que adquirieron los alumnos al apreciar el compromiso e implicación del profesor con cada alumno, y la confianza que depositaba en ellos; También por la recepción de comentarios positivos y negativos, W15: "Me pareció muy interesante el enfoque del profesor al darnos retroalimentación, enfocándose en los aspectos positivos de nuestro trabajo y demostrando que no es trágico haber cometido algunos errores $y$, en cambio, podemos usar esos errores para estar más motivados, aceptar el desafío y mejorar. Esta estrategia de retroalimentación es indudablemente interesante sin duda nos ayudará a mejorar los próximos proyectos sin perder la motivación".

El estudio de la relación entre los estudiantes permitió identificar un valor "intragrupal" en la experiencia, casi siempre relacionado con la diversidad cultural W01: "fue agradable porque éramos un grupo pequeño y nuestras interacciones se hicieron agradables rápidamente. Tuvimos la oportunidad de llegar a conocer a cada uno más profundamente y en consecuencia también pudimos ver las diferencias culturales entre los enfoques de trabajo". Este valor social intragrupal a veces se esperaba, y en otras ocasiones fue inesperado, sorprendiéndoles rasgos positivos de personalidad de los compañeros (apertura, cortesía) que facilitaron el rápido establecimiento de fuertes vínculos entre ellos. 
Gallarza, M.G., Fayos Gardó, T., Fontana Vinat, M., Derqui Zaragozá, B. (2018). Introspección reflexiva del estudiante sobre su experiencia en másteres internacionales. @tic revista d'innovació educativa, 21, 57-66.

La interacción entre los estudiantes, que es uno de los principales resultados positivos de la experiencia investigada, es una forma de producir no solo beneficios sociales, sino también emocionales y funcionales, W14: "Como miembro de un equipo, mejoré mi capacidad para escuchar a los demás y compartir ideas. Veo que el equipo funciona como un excelente ejercicio para los estudiantes. Esta forma de trabajar nos prepara para el futuro y nos da la oportunidad de compartir diferentes opiniones para crear una sinergia en nuestros esfuerzos", lo que proporciona evidencia de la adecuación a la experiencia universitaria de un enfoque integral comprehensivo del valor percibido de consumo (propio de autores como Holbrook, 1999; o Sheth et al., 1991), tal como el que se siguió en este estudio.

Así, de acuerdo con en el enfoque basado en el valor que proponemos, la experiencia descrita puede considerarse integral (Holbrook, 1999), ya que algunas citas fueron una combinación de expresiones cognitivas y afectivas como, por ejemplo, 108: "Estos últimos seis meses han sido para mí un verdadero placer. Esto se debió a varios ingredientes: el espíritu de la clase fue muy agradable, gracias a mis excelentes colegas, los profesores fueron muy interesantes y motivadores, el contenido fue completamente nuevo para mí y realmente disfruté de esta nueva asignatura. Mi nuevo entorno encajó completamente con mis expectativas".

\subsection{Costes}

En cuanto a los costes, el número de citas es muy inferior al de beneficios. La mayoría de los costes citados tiene una naturaleza funcional, y mencionados por estudiantes europeos (principalmente mujeres). Estos costes funcionales se relacionan principalmente con, la falta de tiempo para terminar los trabajos, debido al elevado número de trabajos tanto individuales como en grupo en la asignatura y en otras asignaturas del master, pero también con el deseo de los alumnos de obtener más conocimiento sobre algunos temas específicos, en particular con todos los relacionados con la multiculturalidad y sus diferencias.

Algunos comentarios fueron codificados como costes, aunque podrían considerarse sugerencias, I12: "Creo que si comenzamos desde el principio con un proyecto global de todo el tema y aplicamos todas las herramientas después de cada sesión será mucho mejor". En cambio, sí se mencionaron sugerencias explícitas y, por lo tanto, deficiencias de la experiencia, en relación con el proceso metodológico: W06: "La única sugerencia que estaba dispuesto a hacer en este punto del curso era incluir ejemplos más variados (...) casos más particulares como ejemplos y marcas y compañías menos conocidas". Si contrastamos nuestros resultados con los de investigaciones similares previas (Strauss et al., 2014), sorprendentemente en los nuestros no identificamos quejas relacionadas con las formas de calificar a los estudiantes.

Por último, surgieron algunos costes emocionales, mencionados principalmente por mujeres de la UE y del iMBA, relacionados con la desequilibrada dedicación de tiempo asignada a las presentaciones, W14: "Me gustó mi tema y me decepcionó no presentarlo a los demás como se suponía. La falta de tiempo no nos dio esta posibilidad".

En algunos casos, los estudiantes expresaron su frustración respecto a la finalización de las tareas, lo que ha sido codificado como negativo ya que reflejan sentimientos y emociones negativos: I12: "Al final tendremos un proyecto global en el que trabajamos cada semana paso a paso. Lo disfruté, pero no estaba satisfecho con mi trabajo porque no di todo de mí. Incluso no lo leí todo cuando terminé. Era imposible hacer al mismo tiempo un proyecto global para la asignatura y los distintos pequeños casos.".

Algunas otras declaraciones, con respecto a las oportunidades laborales y la vida profesional, mostraron los dilemas de los estudiantes en cuanto a juzgar determinadas prácticas y enfrentarse con su propia personalidad, especialmente los alumnos, psicólogos del programa WOP-P: W08: "Estoy muy interesado en el marketing, pero tengo algunos problemas "éticos", en el sentido de que creo que si quiero poner mi conocimiento psicológico en el mundo del marketing, tengo que aceptar algún compromiso. Creo que si quiero trabajar en este campo también debería aceptar colaborar con empresas que no respeten los roles éticos".

En general, a diferencia de otros trabajos (Moore y Hampton, 2015), un aspecto muy positivo a destacar es que, entre los costes referidos por los estudiantes, no se hace mención a la incomodidad del trabajo en equipos interculturales, más bien al contrario. Sin embargo, sí se hace referencia a las tensiones internas de los estudiantes con respecto a sus futuras oportunidades de trabajo, las cuales merecen una mayor consideración por parte de las universidades, y especialmente por los profesores de marketing.

\section{Conclusiones}

Este trabajo ha estudiado el contenido de la reflexión introspectiva de los estudiantes respecto de su experiencia universitaria, mostrando la riqueza de la experiencia y discutiendo las reflexiones personales organizadas en forma de dimensiones del valor de la experiencia. Todo ello de acuerdo con la literatura sobre comportamiento del consumidor que entiende el valor percibido como un trade off entre beneficios y costes, resultando más numerosas las dimensiones positivas que las negativas, y pudiéndose además categorizar con relativa facilidad dichas dimensiones según su diferente naturaleza (funcional, emocional y social).

La principal contribución de este trabajo se refiere a la utilidad de la autorreflexión introspectiva de los estudiantes para la enseñanza en nuestras aulas universitarias. Siendo una práctica bastante innovadora e inexplorada en España en la educación superior en general, y sobre todo en marketing, tanto para estudiantes como para profesores, este trabajo ha evidenciado que la investigación reflexiva en general, y el uso de los reflective statements en particular (u otras técnicas cualitativas reflexivas similares como blogs o cuadernos) pueden ser una herramienta para una mejor comprensión de las expectativas y el rendimiento de los estudiantes, lo cual es además crucial en grupos interculturales. Adicionalmente, los resultados también han mostrado algunas pautas para los profesores de Marketing, que pueden entenderse como una contribución adicional interdisciplinar.

En este sentido, de los resultados de este trabajo se desprende que el papel del profesor universitario en este ámbito de metodologías de reflexión activa debe ser proactivo y colaborativo, en aras de una mayor cocreación, y sumarse al que ya tiene como docente e investigador. El reto de integrar en su desempeño un conjunto de conocimientos, habilidades, competencias y valores está claramente en consonancia con su rol primario de docente, esto es, con la idea de "incluir en la 
Gallarza, M.G., Fayos Gardó, T., Fontana Vinat, M., Derqui Zaragozá, B. (2018). Introspección reflexiva del estudiante sobre su experiencia en másteres internacionales. @tic revista d'innovació educativa, 21, 57-66.

enseñanza, acciones que refuercen la dimensión personal de los estudiantes, la autonomía en la gestión del aprendizaje y el desarrollo de actitudes críticas y reflexivas ante la acelerada profusión de información y conocimiento existentes en la sociedad actual" (Moya et al., 2010: p.18), y tanto más para aquellos contextos en los que el estudiante se forma en grupos interculturales donde el componente emocional juega un papel determinante. La función esencial del profesorado en el fomento de la interculturalidad ya es conocida (Ciges, 2012) pero esta puede potenciarse con las técnicas reflexivas, al reflejar éstas actitudes, estrategias e interrelaciones en la tarea educativa. Se necesitaría, por tanto, "un nuevo perfil docente, de un profesorado reflexivo y crítico ante la diversidad cultural" (Ciges, 2012: p.3). Por lo que es recomendable que para estos fines se promueva la idea de un profesorado con una formación intercultural intencionada y formado en metodologías activas reflexivas.

Como todo trabajo empírico, este cuenta con limitaciones, que pueden, en su subsanación dar lugar a líneas de investigación futura. Así, el resultado del desequilibrio en la experiencia entre beneficios y costes (23) debe contrastarse en futuras investigaciones con análisis estadísticos de corte longitudinal, como en Rienties et al. (2014). Además, como una limitación de este trabajo, ya que el ejercicio no fue anónimo, consideramos que pueden haber surgido ciertas respuestas deseables o sesgadas. Es más, el hecho de que sea más fácil para los estudiantes proporcionar retroalimentación sobre hechos funcionales que sociales o emocionales, como se ha constatado por el número de citas, es otra conclusión a tener en cuenta por los profesores cuando planteen el ejercicio de expresar sentimientos y sensaciones sobre el proceso de aprendizaje. Por último, los beneficios y costes de la interculturalidad y su efecto en la autoevaluación realizada por los estudiantes sobre su propio proceso de aprendizaje han sido poco debatidos en este documento, lo que consideramos una limitación. Es bien conocido que considerar las diferencias culturales en la investigación cualitativa es necesario, pero difícil (Nevid y Sta. Maria, 1999). Así, aunque generalizar los efectos de la valoración de las universidades según el país de origen puede plantear algunos problemas, una futura propuesta de investigación, sería combinar la exploración del análisis de contenido realizado aquí a través del software Atlas-Ti con técnicas cuantitativas más sofisticadas. Una aproximación metodológica más completa podría basarse en las llamadas metodologías mixtas, que permitirán explotar todas las posibilidades de este tipo de enfoque doble e integrado (Molina-Azorín, 2016; Harrison y Reilly, 2011) y podría arrojar así una nueva luz, más científica y extrapolable, sobre estos aspectos interculturales vividos por nuestros estudiantes en el aula.

De todos modos, consideramos que la necesidad de adoptar nuevos enfoques para la evaluación de la educación superior en España merece mayor debate, y esperamos que este documento no sólo habrá ayudado a progresar en esta dirección, sino también anime a fomentar el intercambio futuro de experiencias similares.

\section{Bibliografía}

Afshar, H.S. \& Rahimi, M. (2016). Reflective thinking, emotional intelligence, and speaking ability of EFL learners: Is there a relation? Thinking Skills and
Creativity,

19 ,

https://doi.org/10.1016/j.tsc.2015.10.005

Alfaro, I. J., Boullosa, A. P., Andreu - Besó, J. V., De Lamo, J., Sobrado, L., Sanz, R. \& Arias, J. M. (2009). Student opinion of the information policies of four Spanish universities with regard to Erasmus and other international programmes. Higher Education in Europe, 34(3-4), 313-332. https://doi.org/10.1080/03797720903355554

Bain, K. (2006). Lo que hacen los mejores profesores de universidad. Universitat de València.

Bunce, L., Baird, A., \& Jones, S. E. (2017). The student-as-consumer approach in higher education and its effects on academic performance. Studies in Higher Education, 42(11), 1958-1978. https://doi.org/10.1080/03075079.2015.1127908

Ciges, A. S. (2012). La formación intercultural del profesorado: estrategias para un proceso de investigación-acción. Educatio Siglo XXI, 30(1), 113-132.

Dahl, A. J., Peltier, J. W., \& Schibrowsky, J. A. (2018). Critical Thinking and Reflective Learning in the Marketing Education Literature: A Historical Perspective and Future Research Needs. Journal of Marketing Education, DOI: 0273475317752452

De La Fuente Mella, H., Marzo Navarro, M., Riquelme, R., \& Jesús, M. (2010). Análisis de la satisfacción universitaria en la Facultad de Ingeniería de la Universidad de Talca. Ingeniare. Revista chilena de ingeniería, 18(3), 350-363. http://dx.doi.org/10.4067/S071833052010000300009

Denzin, N. K. (2001). The reflexive interview and a performative social science. Qualitative research, 1(1), https://doi.org/10.1177/146879410100100102

Deshpandé, R. (1983). Paradigms Lost: On Theory and Method in Research in Marketing, Journal of Marketing, 47 (Fall) 101-110.

Dewey, J. (1916). Democracy and Education. New York: MacMillan.

Dollinger, M. Lodge, J. \& Coate, H. (2018) Cocreation in higher education: towards a conceptual model Journal of Marketing for Higher Education. DOI: 10.1080/08841241.2018.1466756

Fan, W., Cheung, F.M., Leong, F.T.L. \& Cheung, S.F. (2012). Personality Traits, Vocational Interests, and Career Exploration: A Cross-Cultural Comparison Between American and Hong Kong. Students Journal of Career Assessment, 20 (1), 105-119.

https://doi.org/10.1177/1069072711417167

Finney, T.G. \& Finney, Z. (2010). Are students their universities'customers? An exploratory study. Education+Training, 52(4), 276-291. https://doi.org/10.1108/00400911011050954

Gal, I. \& Ograjenšek, I. (2010). Qualitative Research in the Service of Understanding Learners and Users of Statistics. International Statistical Review, 78 
Gallarza, M.G., Fayos Gardó, T., Fontana Vinat, M., Derqui Zaragozá, B. (2018). Introspección reflexiva del estudiante sobre su experiencia en másteres internacionales. @tic revista d'innovació educativa, 21, 57-66.

(2), 287-296. https://doi.org/10.1111/j.17515823.2010.00104.x

Gallarza, M.G.; Seric, M. \& Cuadrado, M. (2017). Trading off benefits and costs in higher education: A qualitative research with international incoming students The International Journal of Management Education, $\quad 15 \quad$ (3), 456-469. https://doi.org/10.1016/j.ijme.2017.08.001

Gibbs, P. (2007). Marketing and education-A clash or a synergy in time? Journal of business research, 60(9), 1000-1002. https://doi.org/10.1016/j.jbusres.2007.01.025

Gummesson, E. (2005). Qualitative Research in Marketing. Road-Map for a Wilderness of Complexity and Unpredictability. European Journal of Marketing, 39 (3/4), 309-327. https://doi.org/10.1108/03090560510581791

Guolla, M. (1999). Assessing the teaching quality to student satisfaction relationship: Applied customer satisfaction research in the classroom. Journal of marketing theory and practice, 7(3), 87-97. https://doi.org/10.1080/10696679.1999.11501843

Harrison, R. L., \& Reilly, T. M. (2011). Mixed methods designs in marketing research. Qualitative Market Research: An International Journal, 14(1), 7-26. https://doi.org/10.1108/13522751111099300

Haug, G. (2016). La internacionalización de la educación superior: más allá de la movilidad europea. La cuestión universitaria, (6), 20-29.

Holbrook, M.B. (1999). Introduction to Consumer Value and Conclusions. In M.B. Holbrook (ed.). Consumer Value: A Framework for Analysis and Research. London: Routledge, pp. 1-28 \& 183197.

Jackling, B., Natoli, R., Siddique, S., \& Sciulli, N. (2015). Student attitudes to blogs: a case study of reflective and collaborative learning. Assessment \& Evaluation in Higher Education, 40(4), 542-556. https://doi.org/10.1080/02602938.2014.931926

Kaur, A., \& Noman, N. (2015). Exploring Classroom Practices in Collectivist Cultures Through the Lens of Hofstede's Model. The Qualitative Report, 20 (11), 1794-1811.

Kelly, P. (2009). Group work and multicultural management education. Journal of Teaching in International Business, 20(1), 80-102. https://doi.org/10.1080/08975930802671273

Klein, S., Benjamin, R., Shavelson, R. \& Bolus, R. (2007). The collegiate learning assessment: Facts and fantasies. Evaluation Review 31(5), 415-439. https://doi.org/10.1177/0193841X07303318

Klenowski, V. \& Lunt, I. (2008). Enhancing learning at doctoral level through the use of reflection? Assessment \& Evaluation in Higher Education, 33(2), https://doi.org/10.1080/02602930701292795

Leclercq, T., Hammedi, W. \& Poncin, I. (2016). Ten years of value cocreation: An integrative review. Recherche et Applications Marketing, 31(3), 26-60.
Ledden, L., Kalafatis, S.P. \& Samouel, P. (2007). The Relationship between Personal Values and Perceived Value of Education. Journal of Business Research, 60, 965-974. https://doi.org/10.1016/j.jbusres.2007.01.021

Lee, H.J., Lee, J., Makara, K.A., Fishman, B.J., \& Hong, Y-I1. (2015). Does higher education foster critical and creative learners? An exploration of two universities in South Korea and the USA. Higher Education Research \& Development, 34(1), 131146.

https://doi.org/10.1080/07294360.2014.892477

Mena-Marcos, J., García-Rodríguez, M., \& Tillema, H. (2013). Student teacher reflective writing: What does it reveal? European Journal of Teacher Education, $\quad 36 \quad$ (2), 147-163. https://doi.org/10.1080/02619768.2012.713933

Miles, M.B. \& Huberman, A.M. (1994). Qualitative data analysis: An expanded sourcebook, Sage Publications, Thousand Oaks.

Mitchell, E. (2013). Reflective course construction: An analysis of student feedback and its role in curricular design. Education for Information, 30 (34), 149-166. DOI: 10.3233/EFI-130942

Molina-Azorin, J. F. (2016). Mixed methods research: An opportunity to improve our studies and our research skills. European Journal of Management and Business Economics, 25 (2), 37-88. https://doi.org/10.1016/j.redeen.2016.05.001

Moore, P., \& Hampton, G. (2015). 'It's a bit of a generalisation, but...': participant perspectives on intercultural group assessment in higher education. Assessment \& Evaluation in Higher Education, 40(3), 390-406. https://doi.org/10.1080/02602938.2014.919437

Moya, J. L. M., Borrasca, B. J., \& Muñoz, F. I. (2010). La enseñanza reflexiva en la educación superior. Cuadernos de docencia universitaria, 17.

Nevid, J.S. \& Sta. Maria, N.L. (1999). Multicultural issues in qualitative research. Psychology \& Marketing, 16(4), 305-325. https://doi.org/10.1002/

O'Shaughnessy, J. \& Holbrook, M.B. (1988). Understanding Consumer Behavior: The Linguistic Turn in Marketing Research. Journal of the Market Research Society, 30(2), 197-223.

O'Shaughnessy, J. (2009). Interpretation in Social Life, Social Science, and Marketing. Nueva York, USA: Routledge.

Patton, M.Q. (2002). Qualitative Research Evaluation Methods (3rd ed). California: Sage Publications.

Prahalad, C.K. \& Ramaswamy, V. (2004). Co-creation experiences: The next practice in value creation. Journal of Interactive Marketing 18(3), 5-14.

Rafferty, P. D. (2013). Group work experiences: Domestic MBA student experiences and outcomes when working with international students. Journal of Further and Higher Education, 37(6), 737-749.

Rienties, B., Luchoomun, D. \& Tempelaar, D. (2014). Academic and social integration of Master 
Gallarza, M.G., Fayos Gardó, T., Fontana Vinat, M., Derqui Zaragozá, B. (2018). Introspección reflexiva del estudiante sobre su experiencia en másteres internacionales. @tic revista d'innovació educativa, 21, 57-66.

students: a cross-institutional comparison between Dutch and international students. Innovations in Education and Teaching International, 51(2), 130141.

Rivza, B. \& Teichler, U. (2007). The changing role of student mobility. Higher Education Policy, 20(4), 457-475.

Sheth, J.N., Newman, B.I. \& Gross, B.L. (1991). Beyond semiotics and hermeneutics: Discourse analysis as a way to interpret consumers' discourses and experiences. Qualitative Market Research, 11(2), 177-191.

Sinkovics, R. \& Alfoldi, E. (2012). Progressive Focusing and Trustworthiness in Qualitative Research. The Enabling Role of ComputerAssisted Qualitative Data Analysis Software (CAQDAS). Management International Review, (52), 817-845. DOI: 10.1007/s11575-012-0140-5

Sitz, L. (2008). Beyond semiotics and hermeneutics: Discourse analysis as a way to interpret consumers' discourses and experiences. Qualitative Market Research: an international journal, 11(2), 177-191. https://doi.org/10.1108/13522750810864431

Smith, S. (2016). (Re)Counting Meaningful Learning Experiences: Using Student-Created Reflective Videos to Make Invisible Learning Visible During PBL Experiences. Interdisciplinary Journal of Problem-Based Learning, 10(1). DOI: 10.7771/1541-5015.1541

Strauss, P., U-Mackey, A. \& Crothers, C. (2014). They drag my marks down!' - challenges faced by lecturers in the allocation of marks for multicultural group projects. Intercultural Education, 25(3), 229241.

https://doi.org/10.1080/14675986.2014.905361

Swensson, G. \& Wood, G. (2007). Are university students really customers. When illusion may lead to delusion for all. Internacional Journal of Educational Management, 21(1), 17. https://doi.org/10.1108/09513540710716795
Sweeney, J. \& Soutar, G. (2001). Consumer Perceived Value: The Development of a Multiple Item Scale. Journal of Retailing, 77 (2), 203-207.

Tian, M. \& Lowe, J. (2013). The role of feedback in cross-cultural learning: a case study of Chinese taught postgraduate students in a UK university. Assessment \& Evaluation in Higher Education, 38(5), 580-598. https://doi.org/10.1080/02602938.2012.670196

Vargo, S.L. \& Lusch, R.F. (2014). Service-Dominant Logic Premises, Perspectives, Possibilities. Cambridge University Press: Cambridge

Warwick, P. (2014). The international business of higher education-A managerial perspective on the internationalisation of UK universities. The International Journal of Management Education, 12(2), 91-103. https://doi.org/10.1016/j.ijme.2014.02.003

Zeithaml, V.A. (1988). Consumer Perceptions of Price, Quality, and Value: A Means-end Model and Synthesis of Evidence. Journal of Marketing, 52(3), 2-22.

I Cita recomendada de este artículo

Gallarza, M.G., Fayos Gardó, T., Fontana Vinat, M., Derqui Zaragozá, B. (2018). Introspección reflexiva del estudiante sobre su experiencia en másteres internacionales. @tic revista d'innovació educativa, 21, 57-66. https://doi.org/10.7203/attic.21.13299 